\title{
Sachregister / Index of Subjects
}

AfD 25, 86f., 94, 108, 115, 203

Affective Turn 263

Affekt 108-111, 192

Alternative Fakten / Alternative Facts 1f., 4, $13,53,65-82,105,137,145,172$

Anekdote 30, 32, 34ff., 108

Authentizität / Authenticity 5, 8f., 109, 129, 136, 139, 141ff., 242, 250f., 258, 265, 281ff., 287ff., $292 \mathrm{ff}$.

Autobiografie / Autobiography 8, 281ff., 285, 289

Autorität 73, 113, 116, 119, 141 f.

Brexit 5, 9, 14, 48, 60ff., 67, 75, 127f., 130, 146f., 152, 203, 215, 297ff., 312ff.

Bullshit / Bullshitting 27, 91, 105

Cambridge Analytica 4, 48, 53

Conspiracy Theories / Stories

( $\nearrow$ Verschwörungstheorien) 127-156, 285

Corona-Pandemie 6, 127f., 139, 150, 199, 203ff., 210

Cultural Studies 181, 186

Deep Story 4, 65, 72-82, 119, 281, 292f., 294

Desinformation 1, 6, 127f., 131, 191

Desinformationskampagne 199-209.

Emotion / Emotionalisierung / Emotionalität 4, 6, 91, 83f., 93, 158ff., 163, 173, 177, 180, 192ff., 254

Empathie / Empathy 8, 74, $263 \mathrm{ff}$.

Empirie 1, 129, 183, 253, 258

Erfahrung / Experience 104, 220ff., 225, 229, 251, 281, 286ff., 290

Euroscepticism 298f., 302f., 306, 312, 317

Evidenz 15, 34

Fact and Fiction 220f., 281-296

Fact Checking 6, 132, 199, 207, 210
Fake News 1, 7, 49, 53, 105, 128, 135ff., 151, 157, 161, 172, 191, 199ff., 202-209

Fakten 1ff., 13, 15, 40, 90ff., 94, 105, 132, 141, 148f., 159

Faktualität - Fiktionalität 1, 3, 7f., 33, 104f., 116, 119f., 137, 177, 191, 194, 234ff., 243, 245, 258, 281-296

Fehlinformation 106

Fiktion / Fiction / Fictionality / Fictionalization 104, 284ff., 287, 292

Filterblase / Filter Bubbles 134, $178 f$.

Flüchtlingskrise 2015 6, 128, 138

Focalization 272

Frankfurter Schule 98

Glaubwürdigkeit 30

Grand Narrative / Große Erzählung 68, 70, $72,80,215-232$

Halbwahrheit 2f., 23-43

Identitäre Bewegung 39

Identität / Identity $8,76,87,95,108,115$, 158, 171f., 185, 284, 290

- Identity Politics 8, 284, 290, $292 \mathrm{f}$. Imagination 84f., 110, 247f., $257 \mathrm{f}$.

Imagined Community 301

Immersion 251, $255 \mathrm{f}$.

Impeachment 49, 55, 61

Intersectional Theory 291

Kausalität 13-22

Kleine Erzählung 70, 72, 78

Kohärenz 157, 163

Komplexitätsreduktion 36, 40, 105ff., 150f., 174

Konsens $184,186 \mathrm{ff}$.

Kontingenz 40, 92, 96, 106, 112, 114, 118, 120, 150f., 157, 165, 168

Konvergenzkultur 5, 105f., 112ff., 118

Krise 1, 28, 105, 151, 177ff., 194, 207

Kritische Theorie 83,181, 186f., 190

ว Open Access. (C) 2021 Antonius Weixler, Matei Chihaia, Matías Martínez, Katharina Rennhak, Michael Scheffel und Roy Sommer, publiziert von De Gruyter. (c) BY Dieses Werk ist lizenziert unter der Creative Commons Attribution 4.0 International Lizenz.

https://doi.org/10.1515/9783110693065-018 
Lüge / Lies 1, 3f., 13-22., 25, 27, 36, 38, 53, 66, 85, 90, 94, 105, 131, 159, 178f., 191, 234, 282

Macht 161, 166f., 190

Media Literacy 132

Medien 5ff., 28, 90, 112, 127, 129, 133ff., 137f., 144, 148, 160, 163, 172, 177ff., 185f., 189, 194, 199, 234f., 237f., 257

- Breitbart 76

- Compact-Magazin 138

- Fox News 58, 76f., 135, 137

- Medienpolitik 6, 199, 205, 207

- Medientheorie 113f., 117f., 181

- Medium 166, 235, 237, 239f., 243ff., 252f., 258

- RT / Russia Today 137, $202 f$.

- Rundfunk / Fernsehen 138, 178, 201, 205

- Zeitungen / Printmedien 7, 112, 116, 179, 181, 183, 185, 205f., 233f., 236ff., 244ff., 297-321

Meinung (vs. Wissen) 3f., 14, 27, 40, 74, 83, $93,105,159,173,178 \mathrm{f}$.

Memory 221, 282

Metaerzählungen / Metanarrative 69-72

Metafiction 277

Metapher / Metaphor 74, 79, 148, 172, 274 f.

Migration 88, 93, 187

- Migration Narratives 281-296

- Migrationsliteratur 8, 281

Mikronarrationen 70

Motivation 5, 13-22, 31, 149, 129, 149ff., 184

Multiculturalism 286, 289

Mythos / Myth 2, 68, 83, 98, 131, 157, 161, 164ff., 172, 215ff., 225, 227, 229f., $290 \mathrm{ff}$.

- American Dream 74, 77-80, 281, $292 \mathrm{f}$.

- Bayern-Mythos 5, 103-123

- kultureller Mythos 285

- Mythologie 98

- Mythos (Blumenberg) 5, 107, 157, 164f., 168, 173

- Mythos (Cassirer) 158, 160

- Mythos (Barthes) 281, 285

- Mythos (Wirtschaftswunder) 6,169

- Neue Mythologie 39

- politischer Mythos 5f., 158ff., 163, 167, $172 f$.
Narrative 1ff., 5, 7, 9, 13-22, 30f., 33f., 39, 67f., 71, 80, 84, 88, 90ff., 95, 98, 105, 116, 129ff., 134, 136, 138, 145ff., 151, 153

- kulturelle Narrative 9, 68-72

Nationalismus $83 f$.

Neo-liberalism 215-232

Neue Rechte 85

News Literacy 204, $207 f$.

Nonfiction $283 f$.

Öffentlichkeit 1, 6f., 13, 105, 115f., 118, 127, 177ff., 182f., 185ff., 192ff., 200, 203, 238, 246f., 256

Othering 4, 83, 94f., 134ff., 138, 147, 152, 218, 229

Patriotism 300, 309

Plot-Text 109, 112, 114ff., 118ff.

Polemik 6,177, $192 \mathrm{ff}$.

Politik / Politics 1, 3ff., 13, 23, 83, 93, 103f., 106, 151, 159, 187f., 191ff., 236f., 239, 243, 281, 285

- politische Narrative 31, 34, 36, 147

- politische Theorie 93

- politischer Diskurs 47, 83, 103-123, 177, $179,182,186,192 f$.

Populismus / Populism 1, 5, 29, 40, 49, 61f., 83, 85, 88ff., 105f., 108, 114, 130, 148, 203, 294

Postcolonial Melancholia 9, 297f., 302, 304ff., 309, 312

Post-Factual 285, 289

Postfaktisch / Post-Factual 2, 4ff., 9, 13f., 26ff., 40, 47, 66, 73, 83-101, 103ff., 116, 137, 143, 148, 151, 159, 160f., 172, 177ff., 191, 234, 236, 285, 289

Postkoloniale Theorie 95

Postmodern 287

Postmodern / Postmodernism 4, 8, 65, 69ff., $83,90,151,223,263 f f ., 270,277 f .$, $287 \mathrm{ff}$.

Poststructuralism 223

Post-Truth 1, 8, 14, 48f., 53, 61f., 65f., 83, 85, 96, 127, 135f., 151, 157, 264, 297ff., 307f., 314f., 317

Propaganda 86, 114, 131, $201 \mathrm{ff}$. 
Quelle / Quellenkritik 132, 239

Rassismus 204

Realismus 8, 84, 240, 243, 249, $257 f$.

Realist Novel 7, 215-232

Realität / Reality 66, 90f., 95, 114, 116, 152, 162, 264, 307

Science Denial 49

Social Imaginary 216

Source Material 287

Soziale Medien 5, 7, 38, 105, 134, 173, 178f., 190ff., 204, 207

- Facebook 39, 48, 116f., 128, $203 f$.

- Instagram 128

- Telegram 128, 139, 199

- TikTok 199

- Twitter 2, 5, 103-123, 128, 135, 137, 204, 207f., 210

- WhatsApp 128, 199, 210

- YouTube 128, 201, 203

Space $274 f$.

Systemerhalt / Systemvertrauen 29, 182

Tellability 5, 51f., 103, 105, 109ff., 114, 119 Theorie politischer Kommunikation 177ff., 182, 185, 189f., $192 \mathrm{ff}$.
Truth / Truthful / Truthiness 47ff., 55f., 60f., 215-232, 281f., 285, 287ff., $293 f$.

Truth and Reconciliation Commission 4, 49, 62

Verschwörungstheorien ( $\nearrow$ Conspiracy Theories) 5, 20f., 31, 38f., 78, 127-156, 203f., 207

- Anti-Vaccine-Dabatte 72

- Birther-Bewegung 4, 66f., 78

- Reptiloiden-Verschwörung 141, 148

- Reichsbürger 141

- Truther / Truth Movement 31, 128

Wahrheit 1f., 4f., 13ff., 23, 30f., 33f., 38, 40, $71,93,105,120,135,137,178,203,235$, 242, 257

War on Terror 53

Wissen 3, 15, 40, 68, 91, 94, 134f., 138f., 142, 148, 151f., 158, 191

Wissen / Wissenschaft 1, 68f., 72f., 96f., 105f., 119, 129, 131, 149, 189, 191, 234f., 238, 243, 252f., 258 
